\title{
A Survey on Financial Applications of Metaheuristics
}

\author{
AMPARO SOLER-DOMINGUEZ, Universitat Jaume I \\ ANGEL A. JUAN, Universitat Oberta de Catalunya - IN3 \\ RENATAS KIZYS, University of Portsmouth
}

\begin{abstract}
Modern heuristics or metaheuristics are optimization algorithms that have been increasingly used during the last decades to support complex decision making in a number of fields, such as logistics and transportation, telecommunication networks, bioinformatics, finance, etc. The continuous increase in computing power, together with advancements in metaheuristics frameworks and parallelization strategies, are empowering these types of algorithms as one of the best alternatives to solve rich and reallife combinatorial optimization problems that arise in a number of financial and banking activities. This paper reviews some of the works related to the use of metaheuristics in solving both classical and emergent problems in the finance arena. A non-exhaustive list of examples includes rich portfolio optimization, index tracking, enhanced indexation, credit risk, stock investments, financial project scheduling, option pricing, feature selection, bankruptcy and financial distress prediction, and credit risk assessment. The paper also discusses some open opportunities for researchers in the field, and forecast the evolution of metaheuristics to include real-life uncertainty conditions into the optimization problems being considered.
\end{abstract}

- Applied computing $\rightarrow$ Operational research $\rightarrow$ Decision analysis $\bullet$ Applied computing $\rightarrow$ Law, social and behavioral sciences $\rightarrow$ Economics.

Additional Key Words and Phrases: Metaheuristics, Finance, Combinatorial Optimization

\section{INTRODUCTION}

Centered on the wealth creation process, the study of finance and financial instruments is considered to be one of the trendiest disciplines in our modern society. It is thus not surprising that banking, credit, investments, and financial instruments (assets and liabilities) are frequent research topics. Specifically, financial economics has gained an increasing notoriety due to the undeniable impact that micro- and macro-economic factors have on financial decisions and their outcomes. The fast development experienced by the finance-related research brings investors and practitioners around the world countless opportunities. At the same time, this is a field of interest for the academia, since the financial institutions acknowledge the capacity-building and the transfer of knowledge from a plurality of ideas and methods.

Concurrently, as an interdisciplinary area of knowledge, Operational Research comprises a range of problem-solving methods and techniques, which aims at providing efficient solutions to relevant decision-making processes. In this context, optimization methods are recognized as versatile approaches able to solve multifaceted practical problems. For decades, the implementation of optimization methodologies has inspired a broad spectrum of decision-making research that has been applied in different areas, including logistics and transportation, manufacturing and production, healthcare, telecommunication and computing, business and finance, to name just few. Although most optimization methods are well consolidated as

This work has been partially supported by the Spanish Ministry of Economy and Competitiveness (TRA2013-48180-C3-P, TRA2015-71883-REDT), FEDER, and the Universitat Jaume I mobility program (E-2015-36).

Authors' addresses: A. Soler-Dominguez, Finance and Accounting Department, Universitat Jaume I, E12071 Castellon de la Plana, Spain; A.A. Juan, Computer Science Department, Universitat Oberta de Catalunya - IN3, 08018 Barcelona, Spain; R. Kizys, Subject Group of Economics and Finance, Portsmouth Business School, University of Portsmouth, Richmond Building, Portland Street, Portsmouth PO1 3DE, Hampshire, United Kingdom 
powerful mathematical procedures, much less attention has been devoted to tackle tailored problems in applied finance despite the extraordinary development that financial systems, markets, institutions, and assets have been experiencing during the last decades.

In practical financial applications, advances in Operational Research and Computer Science (OR/CS) have brought new solution opportunities. Nowadays, exact methods -relying mostly on different mathematical and constraint programming approaches- are complemented by approximate algorithms -e.g., heuristics and metaheuristics- to tackle near-optimal solutions to complex combinatorial-optimization problems. Although the latter approaches have attracted less attention in the literature of finance so far, the boundaries of these techniques are gaining popularity during the last two decades. Among others, Talbi [2009], Yang [2010], and Gogna and Tayal [2013] provide excellent overviews on these approximate methods and their applications in solving difficult optimization problems. Although approximate methods do not warrant a global optimum, they are able to provide at least near-optimal solutions in reasonable computing times to realistic NP-hard problems, where exact approaches would require an excessive amount of computing time. Gilli et al. [2008] and Gilli and Schumann [2012a] promote the use of heuristic-based approaches. Furthermore, the emergence of matheuristics (integration of exact methods and metaheuristics) is mapping a new scenario where hybrid strategies aim to exploit the benefits of both methodologies [Puchinger and Raidl 2005; Raidl 2006; Jourdan 2009; Blum et al. 2011; Boschetti and Maniezzo et al. 2011; Boussaïd et al. 2013; Serani et al. 2015]. Likewise, combinations of different metaheuristic frameworks are conceived. Recently, Juan et al. [2015] propose the use of simheuristics -integration of metaheuristics and simulation- to solve combinatorial optimization problems with stochastic components. Figure 1 shows the time evolution of journal-published scientific articles that are related to the application of metaheuristics in finance. A growing trend can be clearly observed in this data. Therefore, although a vast number of existing optimization papers in finance makes use of exact methods, it seems evident that the popularity of metaheuristics to support decision making in this area is gaining momentum among researchers -which is reasonable due to the complexity of real-life financial problems. Being an interdisciplinary topic of interest for researchers and practitioners belonging to different communities, the distribution of published articles by knowledge area and journal is depicted in Table I.

This study provides a review of metaheuristic algorithms that are used to solve combinatorial optimization finance-related problems. By doing so, it contributes to the existing literature in different ways. Firstly, it reviews how metaheuristics have been used to deal with classical optimization problems in finance. Secondly, it identifies emerging optimization problems in the field. Thirdly, the paper also discusses how simheuristic algorithms can extend metaheuristics to address financial combinatorial optimization problems considering real-life uncertainty. Overall, our research brings to the attention of financial economists and professionals recent methodological advances that can help seize the opportunity to improve the quality of financial decisions. 
Fig. 1.

N. Articles vs. Year

of related

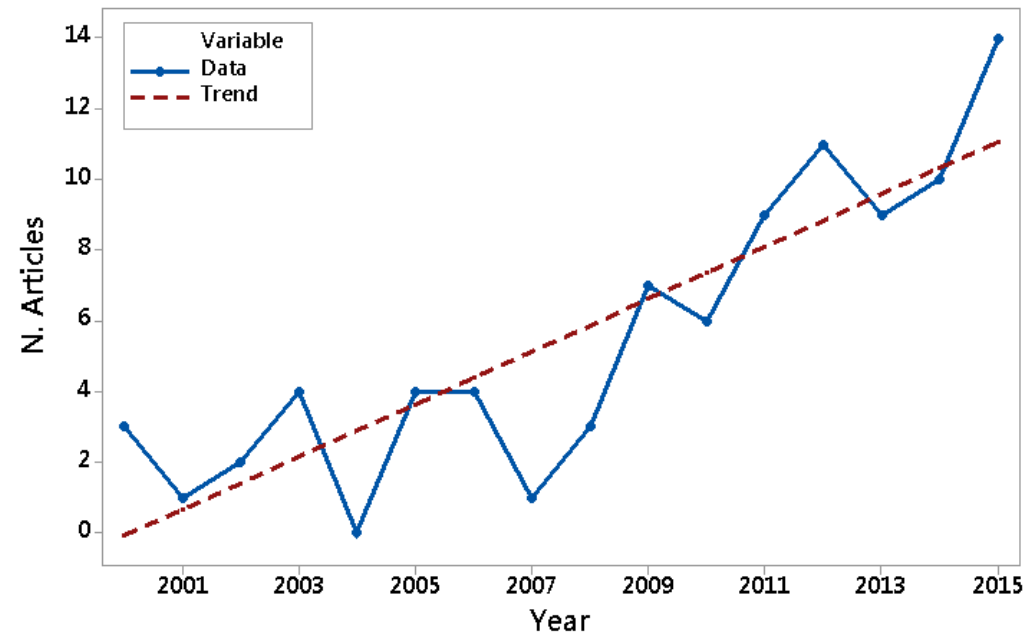

Evolution articles published period

for the

2000-

Table I.

Distribution of articles by knowledge area and journal

\begin{tabular}{|c|c|c|}
\hline Knowledge Area & Journal Title & $\begin{array}{c}\text { \# papers } \\
(2000-2015)\end{array}$ \\
\hline \multirow{9}{*}{$\begin{array}{l}\text { Operational Research \& } \\
\text { Management Science }\end{array}$} & European J. of Operational Research & 18 \\
\hline & Expert Systems with Applications & 15 \\
\hline & Annals of Operational Research & 4 \\
\hline & J. of the Operational Research Society & 2 \\
\hline & OR Spectrum & 2 \\
\hline & Computers \& Operational Research & 2 \\
\hline & Applied Mathematics and Computation & 2 \\
\hline & J. of Scheduling & 1 \\
\hline & J. of Global Optimization & 1 \\
\hline \multirow{4}{*}{ Management } & Management Science & 1 \\
\hline & Computational Economics & 1 \\
\hline & Int. J. of Project Management & 1 \\
\hline & Int. J. of Operational \& Production Management & 1 \\
\hline \multirow{4}{*}{ Finance } & J. of Banking \& Finance & 5 \\
\hline & Quantitative Finance & 1 \\
\hline & J. of Financial Economics & 1 \\
\hline & J. of Risk & 1 \\
\hline \multirow{2}{*}{ Economics } & Economic Modelling & 1 \\
\hline & The J. of Political Economy & 1 \\
\hline \multirow{5}{*}{$\begin{array}{l}\text { Computer Science \& } \\
\text { Artificial Intelligence }\end{array}$} & Applied Soft Computing & 3 \\
\hline & Information Sciences & 2 \\
\hline & Applied Mathematics and Computation & 1 \\
\hline & Nonlinear Analysis: Real World Applications & 1 \\
\hline & J. of Experimental \& Theoretical AI & 1 \\
\hline
\end{tabular}

The remainder of this study is structured as follows. Section 2 provides a brief overview of key metaheuristic techniques for readers with a background different from optimization. Section 3 reviews research that uses metaheuristics to solve classical optimization problems in finance. Emerging optimization problems in finance are discussed in section 4. A short discussion on computational times is provided in Section 5. Section 6 proposes future trends regarding the intensification in the use of some types of metaheuristics, as well as the combination of metaheuristics with simulation to solve stochastic combinatorial optimization 
finance-related problems. Finally, section 7 summarizes the most relevant findings of this study.

\section{A BRIEF OVERVIEW ON METAHEURISTIC ALGORITHMS}

This section provides a brief overview on metaheuristic algorithms for those readers who are not familiar with these optimization methods. For a more detailed review on metaheuristics and their potential applications in different fields, the reader is referred to Talbi [2009] and Luke [2013]. Metaheuristics are optimization algorithms that describe efficient methods and techniques to search for an optimal or nearoptimal solution inside typically vast solution spaces. Usually, these searching methods use some kind of stochastic but guided behavior in order to explore the solution space. Metaheuristic algorithms have gained popularity during the last decades since they can provide near-optimal solutions with reasonable computing times to complex (NP-hard) optimization problems. Metaheuristics are often applied to support real-life decision-making, as they are generally faster than exact methods when solving realistic and large-size versions of NP-hard combinatorial optimization problems [Caceres-Cruz et al. 2014]. Fast computational times of metaheuristics are of key importance in practical applications. Indeed, many managerial and financial decisions have to be reached within a very short period of time, often within few minutes or even seconds.

Metaheuristics can be categorized into single-solution and population-based algorithms (Figure 2). On the one hand, single-solution metaheuristics generate a single solution and then improve it using an iterative searching process inside the solution space. The Greedy Randomized Adaptive Search Procedure (GRASP), Simulated Annealing (SA), Iterated Local Search (ILS), and Tabu Search (TS) are typical examples of single-solution based metaheuristics [Schlottmann and Sesse 2004b]. These algorithms are the ones preferred by researchers with a background in Operational Research, since, at least to some extent, they are more "white-box" methods -i.e., their internal behavior relies on a search strategy that usually follows an easy-to-understand logic. On the other hand, population-based metaheuristics use the information about a complete set of solutions to scan the solution space for good results [Boussaid et al. 2013]. Ant Colony Optimization (ACO), Particle Swarm Optimization (PSO), Artificial Bee Colony (ABC), Firefly Algorithms (FA), and Evolutionary Algorithms (EAs) -which include Genetic Algorithms (GA), Genetic Programming (GP), and Differential Evolution (DE)- are typical examples of population-based metaheuristics. These algorithms are usually developed in Computer Science and Artificial Intelligence, since they usually rely on computing parallelization strategies and require intensive parameter fine tuning (to some extent, they are more "black-box" methods). Another dimension into which metaheuristic algorithms can be classified is local search and constructive procedures. A local search algorithm starts by constructing an initially feasible solution, which is then progressively improved through local changes in the attempt to find local optima close to the initial solution. Unlike local search algorithms, constructive procedures build up a solution iteratively by selecting a promising movement at each step. In practice, both local search and constructive procedures are often combined inside the metaheuristic framework to attain more competitive results. 


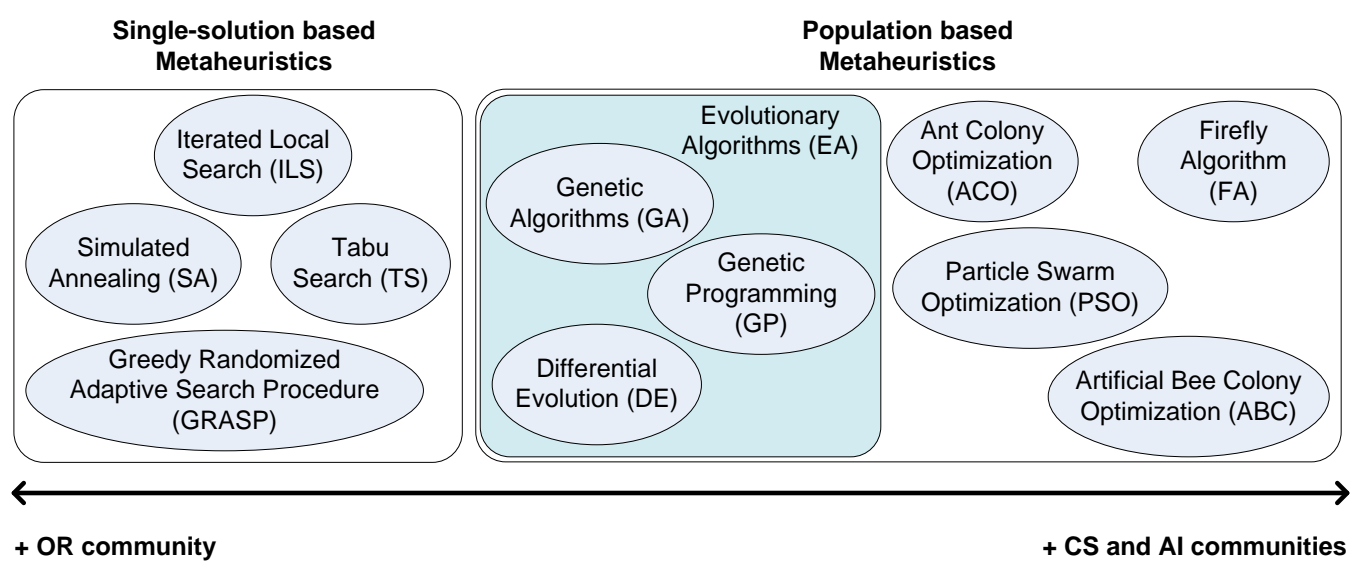

Fig. 2. Classification of key metaheuristics.

\section{CLASSICAL OPTIMIZATION AND SELECTION PROBLEMS IN FINANCE}

This section reviews scholarly articles on applications of metaheuristics, with a particular emphasis on 'classical' optimization problems in finance. These problems are classical in the sense that they have been studied for several decades now. Examples of classical problems include the Portfolio Optimization and Selection Problem, the Index Tracking Problem, the Enhanced Indexation Problem, the Creditrisk Optimization Problem, the Stock Investment Problem, and the Project Scheduling Problem. Table II summarizes the metaheuristic techniques that have been utilized to solve each of these problems.

Table II. Metaheuristic approaches used to solve classical optimization problems in finance

\begin{tabular}{|c|c|c|c|c|c|c|c|c|c|}
\hline & \multicolumn{7}{|c|}{ Single-solution } & \multicolumn{7}{|c|}{ Population-based } \\
\hline Optimization Problem & SA & TS & GA & DE & GP & ABC & FA & ACO & PSO \\
\hline Portfolio Optimization & $\mathrm{X}$ & $\mathrm{X}$ & $\mathrm{X}$ & $\mathrm{X}$ & & $\mathrm{X}$ & $\mathrm{X}$ & $\mathrm{X}$ & $\mathrm{X}$ \\
Index Tracking & $\mathrm{X}$ & & $\mathrm{X}$ & $\mathrm{X}$ & & & & \\
Enhanced Indexation & & & $\mathrm{X}$ & & & & & & \\
Credit-risk Optimization & & & $\mathrm{X}$ & & & & & & $\mathrm{X}$ \\
Stock Investment & & & $\mathrm{X}$ & & $\mathrm{X}$ & & & \\
Project Scheduling & $\mathrm{X}$ & $\mathrm{X}$ & $\mathrm{X}$ & \multicolumn{7}{c}{$\mathrm{X}$} & $\mathrm{X}$ & \\
\hline
\end{tabular}


This summary suggests that (i) the Portfolio Optimization and Selection Problem and the Project Scheduling Problem have been widely studied using a plethora of metaheuristic algorithms, probably because they are strongly correlated with other problems in the field; (ii) Evolutionary Algorithms (including GAs, GPs, and DEs) have been employed in solving all of the aforementioned problems, which is probably due to their flexibility and their capacity to deal with real-life constraints; and (iii) single-solution based metaheuristics, which have shown to be very efficient in other fields, have not been employed so far to solve classical optimization problems in finance.

\subsection{Portfolio Optimization and Selection Problem}

The mean-variance theory of portfolio optimization and selection of Harry Markowitz [Markowitz 1952] posits that investors minimize the risk of investment in a portfolio of assets by optimally allocating the weights to those assets subject to: (i) the minimum required (or expected) rate of return on the investment portfolio; and (ii) the availability of resources for investment. In the Markowitz's portfolio optimization and selection problem (POSP), the risk of portfolio investment is measured by the variance of a portfolio return, which can be thought of as a weighted average of returns (with non-negative weights) on individual assets in the portfolio. The resulting objective function is a quadratic function that is minimized subject to the above constraints. Thus, the POSP deals with a trade-off between minimizing portfolio variance and maximizing expected portfolio return. An advantage of the POSP is that it can be effectively used by the investor to diversify the portfolio risk by investing in a set of assets with imperfectly correlated returns. A disadvantage of the POSP is that -while it is parsimonious and algebraically simple- it underrepresents real-world scenarios. As discussed in Beasley [2013], the problem becomes NP-hard when some realistic constraints are also considered, among others: including bounds on the number of assets allowed in the portfolio, including bounds on the level of investment allowed for each individual asset, and forcing some assets to be mandatory in any portfolio due to the investor preferences. However, the computational intractability can be alleviated by metaheuristic algorithms that provide the decision maker with near-optimal solutions within reasonable computational times.

In this regard, various metaheuristic algorithms have been developed in the related literature. Chang et al. [2000] incorporate the so-called cardinality and quantity constraints in the POSP. The former places a limit on the number of assets that can be selected in a portfolio. The latter sets the ceiling and/or the floor on the weights allocated to each asset in the portfolio. Doering et al. [2016] analyze the role of metaheuristic algorithms in solving the POSP with cardinality and quantity constraints. Specifically, Chang et al. [2000] solve the resulting problem with three different metaheuristic techniques; GA, TS and SA. Since none of the algorithms is found to clearly outperform the others, their recommendation is to execute the three algorithms and combine the results as an efficient way to solve the POSP. Along similar lines, Maringer and Kellerer [2003] analyze the cardinality constrained POSP. Unlike Chang et al. [2000], they solve the POSP by means of using a hybrid metaheuristic that combines SA with evolutionary strategies. Maringer and Kellerer [2003] argue that such a hybrid algorithm is not only efficient but also can be adapted to solve the POSP that features further realistic constraints, including transaction costs, taxes, quantity constraints, alternative risk metrics and return distributions. In an attempt to overview the related literature, Di Tollo and Roli [2008] propose a classification framework for metaheuristic algorithms. They further 
shed light on the potential of hybrid metaheuristics, thus lending support to Maringer and Keller [2003]. They argue that "metaheuristics provide flexible and powerful solving strategies that can effectively and efficiently tackle the various instantiations of the Portfolio Selection Problem, from the basic Markowtz formulation, to more elaborated models including also side constraints". They also recognize the field of financial optimization as an interdisciplinary one, and therefore highlight the necessity of cross-fertilization of ideas between the algorithmic and the finance communities. Cura [2009] proposes a PSO approach for a cardinality constrained version of the POSP. The author uses stock market data on some of the most important financial indices around the world, i.e.: the Hang Seng (Hong Kong), the DAX100 (Germany), the FTSE100 (UK), the S\&P100 (USA), and the Nikkei (Japan). According to the obtained results, the PSO algorithm is competitive relative to other metaheuristic approaches, such as GA, SA, and TS. Soleimani et al. [2009] analyze the POSP with three additional constraints: cardinality constraints, market (sector) value and minimum transaction lots. Their proposed GA is efficient in solving large-size instances of the aforementioned problem.

More recently, Woodside-Oriakhi et al. [2011] use three different metaheuristic algorithms (GA, TS, and SA) to examine the cardinality constrained POSP. In fact, they combine these metaheuristics with exact methods during the solving process, thus creating matheuristics. By comparing with previously published results, the authors show the efficiency of the proposed algorithms with regard to computational time and solution quality.

Gorgulho et al. [2011] propose a GA to manage a portfolio of assets by means of technical analysis indicators. The authors compare the proposed trading strategy with the market itself and a number of other investment strategies. In their experiments they use data from the 2003-2009 periods, which overlaps with the recent global financial crisis. Their results indicate that their approach unambiguously outperforms the other metaheuristics. Di Gaspero et al. [2011] construct a matheuristic algorithm that combines a local search algorithm with quadratic programming to solve a realistic variant of the POSP, which includes minimum cardinality constraints and pre-assignment of assets to the portfolio. Their algorithm finds the optimum in most instances, and is competitive when compared to other novel methods. Golmakani and Fazel [2011] develop a PSO metaheuristic to solve an extended POSP problem with four categories of constraints: cardinality, holdings bounds, minimum transaction lots and sector size constraints. The latter constraint conveniently addresses the investor's belief that investments in more capitalized sectors are less risk that investments in less capitalized sectors. Their findings indicate that the PSO metaheuristic is able to outperform -both in solution quality and computation times- previously developed approaches, especially in largescale problems. Deng et al. [2012] also construct a PSO algorithm to solve the POSP with cardinality constraints. Their algorithm is able to improve the results attained by other existing algorithms, especially for portfolios with low-risk investment. The authors also state their interest in developing new ACO-based metaheuristic algorithms for the POSP. He and Huang [2012] propose an enhanced PSO algorithm for solving four portfolio POSPs with the real data from the Hong Kong stock market. According to their results, their enhanced particle swarm optimization algorithm outperforms other similar approaches. Metaxiotis and Liagkouras [2012] review the existing body of research on POSPs, with a particular emphasis on multi-objective evolutionary algorithms. To this end, they scrutinized 91 scholarly articles in total. Over $80 \%$ of these have come from a two-dimensional approach consisting in the use 
of portfolio's expected return and variance as objectives. Additionally, multidimensional objectives using VaR, annual dividends, expected shortfall, and skewness have been used only to a limited extent. This paper also reviews the constraints alternatives in the formulation of the models, identifying the twoconstraints model as the most popular one. Specifically, they found that cardinality constraints, in conjunction with lower and upper bounds, are the most widely recognized. The authors also put the emphasis on the emerging opportunities derived from the inclusion of additional real-world constraints, which cover more realistic POSPs. Corazza et al. [2013] analyze the POSP considering several alternative measures of risk and penalty functions. They focus on a category of metrics that utilizes information contents in the lower and upper tail of the return distribution. A nonlinear POSP, which takes into consideration several realistic constraints, makes the problem NP-hard. For solving the problem, these authors propose a PSO algorithm. Cesarone et al. [2013] analyze a cardinality-constrained version of the POSP. The authors develop an exact algorithm for solving small size instances of the problem. Their approach consists of picking an asset at the start and then adding one asset at a time in an optimal fashion. Additionally, the authors exploit some theoretical results on quadratic programming. However, they also propose a metaheuristic algorithm for solving larger instances, and employ an exact algorithm for checking the efficiency of the proposed metaheuristic. Lwin and $\mathrm{Qu}$ [2013] investigate an enriched version of the POSP including practical trading constraints such as the cardinality, floor, and ceiling constraints. For solving this problem, they derive a new hybrid algorithm that integrates a DE algorithm with another population based approach. The extended benchmark datasets stored in the OR Library are used to appraise the quality of the new hybrid algorithm. Mansini et al. [2014] comprehensively survey existing models of portfolio optimization. They also review a number of real-world features of POSP problems, such as: transaction costs, transaction lots, cardinality constraints, investment threshold constraints, and decision-dependency constraints. These real-world features comprise the computational approaches -both of exact and heuristic nature- that are used to solve those optimization models. Therefore, considering real-life features has a direct impact in terms of modeling, since some decision variables may be expressed either in relative or absolute values. Furthermore, the authors highlight that the difficulty of the problem is increased when integer or binary variables are included in the model. Di Tollo et al. [2014] define a multi-criteria optimization problem in which both 'active' and 'passive' approaches are combined. An active approach requires the investor to estimate future returns, while in a passive approach the investor aims to construct a portfolio that replicates the behavior of a stock market index. The authors propose an efficient hybrid matheuristic that combines local search and quadratic programming to solve the multi-criteria problem and obtain an approximation of the Pareto frontier. Tuba and Bacanin [2014] present a hybrid algorithm combining two population-based metaheuristics to deal with the cardinality constrained POSP. According to their results, their hybrid approach improves each of the individual approaches and also outperform the ones obtained from GA, SA, TS and PSO. Adebiyi and Ayo [2015] discuss an extended version of the POSP consisting of four categories of constraints: cardinality, expert opinion, holdings bounds, and minimum transaction lots. The authors propose a metaheuristic approach based on an extended variant of the DE algorithm. They also advocate the necessity of introducing expert opinion as an additional constraint to existing portfolio selection models. According to their results, their approach outperforms other metaheuristics methods, including existing GAs, SA, TS and PSO. 


\subsection{Index Tracking Problem}

Index tracking is a buy-and-hold trading strategy, which seeks to passively mimic the behavior of the stock market index with a reduced number of stocks. The index tracker fund is constructed to match the components of a market index. The objective of the index tracker fund's manager is to obtain the same return as the one attained on a market index, such as the S\&P 500. Thus, the index fund strategy represents a relatively stable and efficient vehicle of investment. Indeed, the index tracking funds' popularity lies on their simplicity and readiness to offer a controlled risky option to deliver market-driven returns. The Index Tracking Problem (ITP) aspires to reproduce the performance of a stock market index, albeit without acquiring all of the constituents of the stock market index. Although the ITP is wellknown in finance, it has received scarce attention in comparison to a closely related POSP. There are two stages involved in the index tracking construction: firstly, the selection of stocks problem; and secondly, the determination of optimal weights for the selected stocks. Therefore, the ITP with a partial replication strategy can be solved by applying a metaheuristic approach, and reasonable solutions are obtained in a proper runtime. Also a full replication method is conceived meaning that the same weight proportion is held as represented in the benchmark index. In this case, however, the transaction costs associated with the periodically re-weighting process (necessary to accurately replicate the objective index) are deducted from the index tracker fund performance. Therefore, partial replication is commonly assumed, where minimizing the tracking error (namely the wedge between returns on the tracking portfolio and on the underlying index) is usually considered as the objective function.

In the context of this problem, a number of metaheuristic approaches have been proposed: Beasley et al. [2003] present a population-based evolutionary algorithm specifically, a population heuristic- to solve the ITP. Their analysis attributes central importance to the underlying transaction costs and constraints when applying a partial replication. Reduction tests and results obtained from five data sets are encouraged to be used in further studies. Derigs and Nickel [2003] employ a SA algorithm, and they present their analysis considering a case study on tracking error minimization for a specific German index. Limited computational results are presented when reproducing the benchmark, but authors highlight the reasonable time and quality of the decision support system proposed. Oh et al. [2005] propose a GA to solve an ITP variant. They carry out several experiments and conclude that the proposed GA leads to a notable improvement in the performance of an index, mainly in a more volatile market stance. Rafaely and Bennell [2006] also apply a GA and compare its performance with quadratic programming, which is commonly proposed as the solution approach. Both methods use simulations in order to track their performance by using data on the FTSE100) index. It is concluded that the GA shows an improved quality when appraising the errors in maximizing returns on the index. Krink et al. [2009] suggest the hybridization of DE and combinatorial search to solve the ITP. The problem consists of minimizing the tracking-error volatility with regards to a stock market index. To this end, they implement an evolved DE version, which is able to generate accurate and robust solutions. To solve the ITP, Ruiz-Torrubiano and Suarez [2009] develop a hybrid optimization algorithm that combines a GA with quadratic programming. This hybrid algorithm yields near optimal solutions when it is applied to both synthetic and real world problems. Although the transactions costs are not considered in their analysis, the authors highlight the importance of their inclusion for further research. 
More recently, Guastaroba and Speranza [2012] introduce an advanced heuristic algorithm, referred to as Kernel Search, in order to identify a subset of variables under mixed-integer lineal programming to deal with two variants of the ITP. Transaction costs are included in both optimizations models. Computational results show the effectiveness and efficiency of their proposed approach. Scozzari et al. [2013] develop a DE approach. Their results highlight the appropriateness of heuristic performance to deal with large-size problems. Specifically, the authors emphasize the boundaries of merging exact algorithms with heuristics to address the ITP. They propose the hybridization of heuristic with exact solvers in the following manner: within a large-size multi-period ITP, they propose to use a heuristic algorithm to generate a reasonable good solution for the first period within a short computational time. Then, they use the exact method to determine optimal solutions for all succeeding periods. Chiam et al. [2013] develop a multiple-objective EA to address the implementation of various real-world issues regarding the ITP. They formulate a realistic approach with stochastic capital injections, balanced transaction costs, and additional constraints. The authors make use of out-of-sample data sets. Their analysis is extended to cover time-varying market conditions that affect the index fund during the investment horizon. To test the performance of this approach various rebalancing scenarios are considered and, finally, the multi-objective evolutionary index tracking optimizer (MOEITO) is validated. Finally, Fastrich et al. [2014] propose to use a hybrid DE algorithm to solve the ITP under cardinality and other realistic constraints. These authors employ a financial data set to highlight the complexity of real-world ITPs.

\subsection{Enhanced Index Tracking Problem}

Closely related to the aforementioned ITP, the Enhanced Index Tracking Problem (EITP) is considered a trading strategy that seeks to outperform the returns of the benchmark index. The idea is to mimic the performance of a market index while exceeding the return on the underlying index. Thus, the EITP maximizes the excess return while keeping the tracking error bounded and assuming the same level of risk. Accordingly, the main discrepancy between the ITP and the EITP lies on the active component that the latter approach incorporates: the EITP let the managers and investors to employ active strategies in order to offset the proportion of tracking error that arises mainly from transaction costs. Despite the fact that their approach is of exact nature (Mixed Integer Programming), it is worthy to underscore the seminal work of Canakgoz and Beasley [2009], who consider the traditional ITP but also address the EITP. In particular, their constructed EITP incorporates a semi-active and risk-controlled investment strategy. They carry out an exhaustive literature review in which the ITP and the EITP are treated separately. These authors provide a set of data benchmarks, available from the OR-Library, in an attempt to standardize the data selection process and to compare among different solving approaches. In their approach, the authors envisage limits on the number of stocks and on the total transaction costs as real-world constraints. The computational times are considered to be reasonable, at least for small-size instances.

More recently, Li et al. [2011] propose an evolutionary multiple-objective algorithm, coined as the Artificial Immune System (AIS), to solve the EITP. These authors use the data set proposed by Canakgoz and Beasley [2009]. Although the results are somewhat inconclusive, their metaheuristic algorithm shows a seemingly superior performance. Chavez-Bedoya and Birge [2014] propose a parametric approach to solve the EITP. Although non-linear and non-convex objective functions are easily incorporated into the proposed approach, the selection of appropriate stock 
characteristics in the parametric model is difficult to handle. Authors recommend choosing strategies that mix different typologies of assets. Such strategies aim to avoid excessive exposures to a particular sector or a group of stocks. The advantage of facilitating more information to investors about the portfolio holdings is highlighted, since the optimization is performed over portfolio strategies. Finally, Li and Bao [2014] present a multi-objective algorithm with one-period investment horizon. Authors leave for further research the multi-stage optimization algorithm which characterizes the tracking process control.

\subsection{Credit Risk Management}

Credit Risk Management (CRM) is associated with the constructing and rebalancing of a portfolio of credit assets in order to achieve a desired overall risk level. From a strategic point of view, the risk manager should distribute the credit risk among a diversified set of products. In the first step, the optimization algorithm initially aims at assigning the optimal weight for each asset in the portfolio. In a second step, the optimal risk allocation is determined. The trade-off in this framework is to optimize the credit assets allocation against decreasing the overall risk of investment. In this scenario, the bank acts as an investor, whose goal is to balance the risk and return relation within a portfolio of loans. Mausser and Rosen [2001] discuss the disadvantages of CRM. On the one hand, CRM builds on a large number of scenarios required to model credit events. On the other hand, CRM encounters difficulty in the treatment if risk measures. These authors consider Linear Programming models to minimize risk and trade off risk and return. They also propose a heuristic approach that considers different credit risk measures in CRM. Frey and McNeil [2002] propose value-at-risk $(\mathrm{VaR})$ and compare it with other risk measures to estimate the loss of credit portfolio. VaR is a statistical framework used to appraise the level of financial risk within a bank, company or portfolio of securities over a specific unit of time. VaR is generally parameterized into the value of loss, the probability of that value of loss, and the time span over which the loss takes on that value. Schlottmann and Seese [2004a] highlight again the aforementioned difficulty arising when modeling the CRM as asymmetric loss distributions appear. The authors propose a hybrid EA to solve the risk-return trade-off of credit portfolios. Their approach considers real-life constraints, such as the level of capital constraint. The results show higher quality solutions in comparison with the non-hybridized evolutionary version in terms of speed and convergence. This notwithstanding, one of the weaknesses of the VaR measure is that -while it builds on a sound statistical approach-it fails to assess the scale of losses should they transcend the VaR. In this regard, Yamai and Yoshiba [2005] illustrate how the tail risk of VaR can become problematic for a concentrated credit portfolio. In their research, the optimal solution of a utility maximization problem with VaR constrains is an investment in securities with a non-negligible probability of a large loss that exceeds the VaR level. This is the case when the probability density function of losses presents significant departures from normality. Thus, conditional value-at-risk (CVaR) -that characterizes the average value that is lost over a specific time frame, should the loss surpass the VaR threshold- appears to be a superior measure for risk of any loss distributions as also highlighted in Rockafellar and Uryasev [2000, 2002].

More recently, Iscoe et al. [2012] present optimization techniques that rely on conditional distribution approximations and variance reduction. The results show a reduction in the size of a problem and an improvement in the out-of-sample quality solution. They apply a heuristic algorithm to minimize both VaR and expected 
shortfall while various approximations to the conditional portfolio loss distribution are considered. Lu et al. [2013] propose to solve CRM by applying a novel two-level PSO (TLPSO) algorithm. The objective is to optimize the maximum value of expected loss from a credit portfolio with a budget constraint for the consulting cost. The TLPSO shows a superior performance compared to a GA and a simpler PSO. Zopounidis [2015] highlight the importance of credit risk modeling since the 2008 global financial crisis. Some of the innovations in financial instruments employed by the banking supervision authorities, along with a new regulatory environment, aim to mitigate and prevent the adverse effects of financial instabilities. In this scenario, credit rating appears to be key element within the CRM problem. These authors employ a multiple-criteria decision approach to construct models that predict credit ratings. Dostál [2013] highlights the growing importance of soft computing methods -fuzzy logic, neural computing, evolutionary computation, machine learning, and probabilistic reasoning-in risk management, among other areas of finance.

\subsection{Asset Selection and Market Timing}

Selection of stocks and prediction of the stock market pose serious challenges to asset managers and investors, who seek to identify and select not only the best performing stocks but also the optimal timing to engage in buying or selling those stocks. The stock market also represents an alternative source of funding that companies may use, which means that the stock market contains a large variety of stocks, with varying levels of volatility. All in all, the stock market is considered a powerful vehicle of wealth creation, which challenges investors to decide on the optimal stocks allocation so as a desired trade-off between risk and return is attained. Crucially, this decision must be also encompassed with a sophisticated strategy that aims to outperform the market benchmark. Thus, one of the main concerns related to stock market investment consists of determining the most efficient combination of assets that is at least as profitable as the market index. The solution to this Stock Selection Problem (SSP) is to build up an optimal portfolio of $n$ assets by considering both the expected returns and risk that the portfolio is exposed to. A closely related problem that also has attracted attention since late 80s is the Market Timing Problem (MTP), which refers to the determination of the optimal investment timing (when to buy or sell stocks) assuming that prices change over time. A common procedure to deal with this problem involves designing automated trading rules that dictate where to invest all the available capital (i.e., either in a financial asset or in a risk-free security) within a trading day. Some attempts have been made to develop efficient metaheuristic approaches for both problems. In this regard, Allen and Karjalainen [1999] apply a GA to a broad stock market index (S\&P 500) using daily prices. These authors discuss the advantages that GAs offer, including solutions for technical trading rules. Although little evidence of economically significant technical rules is found, the methodological contribution of this research could be extended to learn or extract some trading rules from other variables as the ones included in the GA are not necessarily optimal. Lee and Jo [1999] support the use of GAs as a proper method able to solve the complexity inherent to the MTP. Iba and Sasaki [1999] utilize a GA to predict stock returns under a stock-picking strategy. This strategy acts as follows: (i) it picks the best-performing stocks; (ii) it determines the number of stocks to buy or sell; and (iii) it determines the timing of buy and sell trades. Also, their paper claims the effectiveness of metaheuristic approaches based on GP. Kim and Shin [2007] study the effectiveness of a hybrid approach that combines the adaptive time delay neural networks and the time delay neural networks with GAs in detecting temporal patterns for stock market prediction tasks. 
More recently, Kaucic (2010) proposes a GA to deal with the MTP. This approach scrutinizes a broad body of technical trading rules in conjunction with a learning method and for different states of the economy. As a result, advantages in terms of robustness in the outcomes achieved and simplicity of the method are heralded from the statistical and economical perspective. Manahov et al. [2014] seek to forecast the equity market behavior for stocks with different capitalization by means of a GP learning algorithm. Their proposed method is shown to outperform a buy-and-hold strategy, and it is found that the constructed forecasts predict better the prices of small rather than large stocks. Chen and Wang [2015] combine a GA with a risk model that aims at building up portfolios. The effective risk attitude is computed taking into account not only the investor risk aversion but also the fluctuating market conditions (bull market vs. bear market). Stock prices are forecast by hybridizing evolutionary computation with CVaR. Manahov et al. [2015] propose another algorithm based on GP to predict stock market returns. Its performance is compared with that of several market indices (S\&P 500, among others). Kaboudan [2000] investigates GP forecasts of stock returns and prices. While stock market returns are unpredictable -as dictated by the efficient market hypothesis- this study finds evidence of GP-predictability of stock prices. Hsu [2011] combines selforganizing map (SOM) with GP to carry out experimental predictions of the finance and insurance sub-index of TAIEX (Taiwan stock exchange capitalization weighted stock index). Hsu's [2011] research findings show that the SOM-GP procedure generates accurate forecasts. Mousavi et al. [2014] develop a multi-tree GP forest approach for dynamic portfolio trading with transaction costs in the stock market of both developed (e.g., Toronto Stock Exchange) and emerging (e.g., Tehran Stock Exchange) countries. Numerical experiments performed by Mousavi et al. [2014] demonstrate that the proposed model significantly outperforms other traditional portfolio selection models in terms of portfolio return and risk adjusted return.

Sheta et al. [2015] build on a multi-gene GP model to derive a prediction model for the S\&P 500 stock market index. Using a number of metrics of prediction accuracy, they find that the GP model generally performs better than a regression model. Berutich et al. [2016] adopt a robust GP approach to develop profitable trading rules for a portfolio of stocks from the Spanish market. Dabhi and Chaudhary [2015] propose a hybrid GP algorithm (Postfix-GP) and wavelet for financial time series prediction. Some promising results are obtained for the stock indexes analyzed (NASDAQ and NSE).

Notably, investment in a portfolio of international stocks requires foreign exchange to be purchased or sold. The quest for optimal investment decisions in the foreign exchange market has spurred a body of research on the use of metaheuristics for currency trading and prediction of exchanges rates. Neely et al. [1997] propose a GP approach to identify an optimal set of currency trading strategies. They find evidence of economically significant out-of-sample returns (net of transaction costs) on trades that involve six exchange rates -USD/DEM, USD/JPY, USD/GBP, USD/CHF, DEM/JPY, and GBP/CHF- over the period of 1981-1995. Mendes et al. [2012] adopt a GA that aims to optimize a set of technical trading rules using two currency pairs: EUR/USD and GBP/USD. They find limited evidence of positive performance in the out-of-sample test series, when transaction costs are taken into account. Along similar lines, Ozturk et al. [2016] optimize a set of technical currency trading rules on two exchange rates: EUR/USD and GBP/USD, albeit in three different time frames. In addition to a GA, as in Mendes et al. [2012], Ozturk et al. [2016] also use a greedy search heuristic. In their experiments -that do not consider 
transaction costs- profitable trades average 60\%. Deng et al. [2015] construct technical trading rules through a hybrid prediction and learning method that combines multiple kernel learning for regression and a GA on three exchange rates, USD/JPY, EUR/USD, and GBP/USD from 2008 to 2011. The proposed method lead to consistently good profits and Sharpe ratios. Dymova et al. [2016] propose a currency trading expert system based on a set of new technical analysis indicators and a new approach to the rule-base evidential reasoning. Using four currency pairs, $\mathrm{EUR} / \mathrm{USD}, \mathrm{GBP} / \mathrm{USD}, \mathrm{EUR} / \mathrm{CHF}$, and USD/CHF, the proposed currency trading system shows a positive performance.

\subsection{Project Scheduling Problems}

The Financial Project Scheduling Problem (FPSP), also known as the Capital Constrained Problem, is an optimization problem considered of crucial importance to support financial decisions under the assumption of certainty. The problem defines a number of activities that require scheduling. It also allows certain interdependences to occur among these activities in the form of precedence constraints. The goal is to identify a schedule which optimizes a suitable objective function. Usually, there are three main variants to consider: (i) project scheduling with time-dependent costs; (ii) project scheduling with constrained resources; and (iii) project payment scheduling with constrained capital. In the first variant, the total expected cost-time minimization is established as the objective function. In the second variant also the capital or resource constraint is required to process an activity. As resources are limited, additional restrictions emerge to schedule the activities. In this context, machine scheduling model seems to be a suitable choice. Indeed, the objective function aspires to determine a schedule that minimizes makespan (time until the last activity is completed). Finally, the last variant's objective consists of assigning modes for activities and payments so that the net present value (NPV) under the of capital availability constraint is maximized. In a review of Icmeli et al. [1993], three related project scheduling problems are described: the project scheduling problem with constrained resources, the time and cost trade-off problem, and the payment scheduling problem. The use of metaheuristic algorithms allows the inclusion of multifactor levels for a number of project characteristics [Smith-Daniels et al. 1996]. Thus, surveys offered by Hartmann and Kolisch [2000] and Kolisch and Hartmann [2006] on the heuristic and metaheuristic applications to the exploration of positive and negative cash flows that investment projects generate.

More recently, Hartmann and Briskorn [2010] provide a comprehensive survey on extensions and modifications of the FPSP with constrained resources. MontoyaTorres et al. [2010] present a GA that solves the resource-constrained FPSP with constrained resources. In Akbari et al. [2011] an ABC algorithm to solve the same problem is proposed. He et al. [2012] address a project payment scheduling problem with capital constraints. They propose a TS algorithm as well as a SA algorithm. Zhou et al. [2013] and Wu et al. [2014] provide an in-depth overview of the recent developments that these problems have experienced. Rahmani et al. [2015] use Differential Search to solve the FPSP with constrained resources. Finally, He et al. [2015] propose a TS and a SA as solvers of the multi-modal NPV problem with constrained resources (also known as multi-modal project payment scheduling problem with capital constraints), which is maximized by optimally assigning activity modes. 


\section{EMERGING OPTIMIZATION PROBLEMS IN FINANCE}

This section reviews scholarly articles that center on applications of metaheuristics to the solving of emerging optimization problems in the financial arena. These problems include the Option Pricing Problem, the Feature Selection Problem, the Bankruptcy Prediction and Financial Distress Prediction Problems, and the Credit Risk Assessment Problem. Table III summarizes the metaheuristic solvers of the above problems. From this table, the following conclusions can be derived: (i) these emerging problems have been less analyzed than the classical ones, and the metaheuristics more frequently employed so far have been ACO, PSO, and GA; and (ii) again, with the exception of TS, single-solution based metaheuristics have not been employed so far despite the efficiency and relatively simplicity of these approaches.

Table III. Metaheuristic approaches used to solve emerging optimization problems in finance

\begin{tabular}{|c|c|c|c|c|c|c|c|c|c|}
\hline \multirow[b]{2}{*}{ Optimization Problem } & \multicolumn{2}{|c|}{ Single-solution } & \multicolumn{7}{|c|}{ Population-based } \\
\hline & SA & TS & GA & $\mathrm{DE}$ & GP & $\mathrm{ABC}$ & FA & $\mathrm{ACO}$ & PSO \\
\hline Option Pricing & & & $\mathrm{X}$ & & & & & $\mathrm{X}$ & $\mathrm{X}$ \\
\hline Feature Selection & & $\mathrm{X}$ & & & & & & $\mathrm{X}$ & $\mathrm{X}$ \\
\hline $\begin{array}{c}\text { Bankruptcy \& Financial } \\
\text { Distress Prediction }\end{array}$ & & & $\mathrm{X}$ & & & & & $\mathrm{X}$ & $\mathrm{X}$ \\
\hline Credit Risk Assessment & & $\mathrm{X}$ & $\mathrm{X}$ & & & & & $\mathrm{X}$ & \\
\hline
\end{tabular}

\subsection{Option Pricing Problem}

Options are derivative instruments of investment that are used for speculative or hedging activities in financial markets. An option derives its value according to their underlying asset values. Understanding the future course of the underlying asset's price and a decision to whether or not enter into an option contract are only two of the multiple challenges that investors experience. Due to the large varieties and volumes of options traded in increasingly competitive derivatives markets, the profitability of option trades to the investors -buyers and sellers of options-can be a daunting task. Option pricing is notoriously difficult to measure due to the high volatility of prices and the dynamism of financial markets. In this context, financial modeling has played a crucial role. Since Option Pricing Theory set the tenets of determining how options should be valued, rich spectrums of alternative innovative models have emerged to cover this topic. Extensions to the Black and Scholes [1973], and Merton [1973] models have been proposed to address their limitations, as their assumptions of a constant volatility and of normally distributed prices neglect reality. Indeed, volatility, time, and dynamism of market conditions are considered key factors in options pricing. A solution for the Option Pricing Problem (OPP) comprises numerous price nodes. The goal is to select the optimal node to exercise the option. Different metaheuristic solvers have been considered to solve the OPP. For instance, Yin et al. [2007] propose an adaptive version of a GA that modifies its internal performance according to the dynamic running of results. Kumar et al. [2008] propose an ACO algorithm for pricing options, and they find that the pricing process is enhanced in comparison with the traditional binomial lattice method.

More recently, Gilli and Schumann [2012b] have studied calibrating option pricing models with the aim of finding parameters that gain consistency between the model prices and the market prices. In their experiments, they use both the DE and PSO 
metaheuristics. Finally, Sharma et al. [2013] and Thulasiram et al. [2014] use PSO to price options.

\subsection{Feature Selection Problem}

In many financial applications, it is crucial to refine the data in order to avoid redundant features and the perverse effect caused by the curse of dimensionality. The Feature Selection Problem (FSP) consists of selecting a small subset of features among those available in a data set- that constitutes the 'optimal' representative features subset. Thus, the goal of the FSP is to identify a subset of factors that can be utilized to perform classification in an optimal fashion. The feature selection seeker is commonly challenged to identify the right method to solve the FSP. In this regard, it is paramount to ensure that only relevant features for classification are selected. Some financial areas in which the FSP has been applied include: credit approval, securities trading, product selection, risk estimation, and corporate bankruptcy. The FSP is characterized as a NP-hard problem, which makes the use of metaheuristic approaches indispensable whenever the dataset is large. Accordingly, Casado [2009] presents an application of the FSP to the selection of financial ratios. He proposes three different metaheuristic strategies: a GRASP algorithm, a TS algorithm, and a memetic algorithm. His experiments demonstrate that both the GRASP and the TS outperform other previous approaches based on the use of GAs.

Similarly, Unler and Murat [2010] discuss how classification has found applications in various financial domains, such as credit approval. They develop a modified discrete PSO algorithm for the FSP. The PSO is then contrasted with other methods. The efficiency of the PSO algorithm is asserted using two metrics, the classification accuracy and the computational time. Xue et al. [2014] also propose a PSO algorithm for solving the FSP. Likewise, Elhedhli et al. [2014] analyze the classification of models to predict the outcome of early stage ventures, and propose two different methods to deal with the aforementioned problem: the Benders decomposition (an exact method) and a TS algorithm. Their results obtained corroborate the efficiency of the TS algorithm for solving both the scoring and classification problems. Finally, Moradi and Rostami [2015] provide a novel refined method of selection based on ACO. Most of the aforementioned papers also make use of financial datasets in their computational experiments.

\subsection{Bankruptcy Prediction and Financial Distress Prediction}

Bankruptcy normally occurs when debtors are unable to pay off their debts. Certain bankruptcy cases are indicative of financial failures that represent symptoms of economic downturns. When bankruptcies become widespread and contagious the impact is disastrous and there are both economic and social losses to financial and non-financial companies, governments, and the overall economic system. Since the 2008's financial crisis, the vulnerability of companies and governments to economic shocks has risen. It is therefore crucial for expert systems to anticipate any event that threatens financial stability. A large body of empirical research has centered on prediction of corporate default, and different methodological approaches have been used to solve this problem. Within these studies, Varetto [1998] highlights the effectiveness of GAs for insolvency prediction, wherein a quicker solution from discriminant analysis can be obtained. Along similar lines, Shin and Lee [2002] propose the use of GAs to predict the failure of corporations by using past financial data. In a similar fashion, in Kim and Han [2003], bankruptcy decision rules are determined with the use of GAs and the input of experts' qualitative opinions. They conclude that GAs can outperform alternative metaheuristic solvers (e.g.: neural 
networks and inductive learning methods) and offer closer results to the experts' problem-solving knowledge. Adnan-Aziz and Dar [2006] present a review of literature and a comparison of methods traditionally employed to tackle this problem, e.g.: statistical tools, artificial intelligence and expert systems, and theoretical approaches. However, these surveys lack the presence of heuristic methods that have been used in several ways already. Similarly, in Kumar and Ravi [2007], the authors present a comprehensive literature review on the Bankruptcy Prediction Problem (BPP), which is closely related to banks and firms. The period analyzed runs from 1968 to 2005, and different predictive methods are analyzed.

More recently, Chen et al. [2011] illustrate the integration of GAs and learning vector quantization in bankruptcy forecasting. By using a real-world data set they conclude that their proposed approach increases effectiveness as features are reduced without adversely affecting the prediction performance. Sun et al. [2014] report a detailed survey which includes a full summary, analysis, and evaluation of financial distress prediction. References included therein discuss insights and interesting applications of GAs, PSO algorithms, and ACO methods as potential alternatives for solving the PPP. Additionally, this study contextualizes the FSP in the existing body of research. Finally, Aruldos et al. [2015] also propose a GA to forecast bankruptcy with qualitative variables.

\subsection{Credit Risk Assessment Problem}

Credit risk is a well-recognized topic in the banking industry. However, the Credit Risk Assessment Problem (CRAP) has gained popularity during the last years. Indeed, it is considered a milestone not only for financial institutions (credit approval), but also for non-financial companies that act as (trade) credit suppliers to their customers, as well as for investors. Credit analysts process large volumes of financial data of companies on daily basis. They are challenged to evaluate the risk of credit and eventually make important decisions on the funding of companies. As Marinakis et al. [2008] argue, corporate and financial credit risk assessment consists of the evaluation of default probability on a loan, and potential future benefits and costs of a loan. They propose three metaheuristic algorithms, TS, GA, and ACO, to solve the feature subset selection problem, which ultimately classifies firms into different credit risk categories according to a reduced number of features. More recently, Oreski and Oreski [2014] develop a hybrid GA that consists of selecting features within a CRAP.

\section{COMPUTATIONAL ISSUES}

When application of these algorithms is considered in a real-world context, computational efforts can play a key role. Unfortunately, not all the reviewed papers provided clear information regarding the processing power employed in their experiments, the size of the instances being solved, and the required execution times. However, several insights and general trends can be grasped from the available data, which are summarized in Table IV. Firstly, improvements in CPU characteristics are associated with lower computational times. In fact, most of the works use reasonably low computing times -in the order of seconds or a few minutes-, to solve instances of realistic size. Secondly, Sharma et al. [2013] and Thulasiram et al. [2014] already use modern graphical process units (GPUs), which benefit from massive multi-thread parallelization of a computational task. Indeed, these GPUs can help achieve significant reductions in the execution times, often to just a few seconds even for complex problems, such as option pricing. 
Table IV. Summary of computational times reported in the literature

\begin{tabular}{|c|c|c|c|c|c|}
\hline Problem & Article & $\begin{array}{l}\text { Solving } \\
\text { Approach }\end{array}$ & CPU & $\begin{array}{l}\text { Problem } \\
\text { Sizes }\end{array}$ & $\begin{array}{l}\text { Execution } \\
\text { Times (s) }\end{array}$ \\
\hline \multirow{13}{*}{$\begin{array}{l}\text { Portfolio } \\
\text { Optimization }\end{array}$} & Chang et al. (2000) & GA / TS / SA & $100 \mathrm{MHz}$ & $31-225$ & 74 to 1964 \\
\hline & $\begin{array}{l}\text { Maringner and Kellerer } \\
(2003)\end{array}$ & $\begin{array}{l}\text { SA + } \\
\text { Evolutionary }\end{array}$ & $900 \mathrm{MHz}$ & $30-96$ & 2 to 45 \\
\hline & Cura $[2009]$ & PSO & $2.1 \mathrm{GHz}$ & $31-225$ & 34 to 919 \\
\hline & Soleimani et al. [2009] & GA & $2.8 \mathrm{GHz}$ & $500-2000$ & 695 (avg.) \\
\hline & $\begin{array}{l}\text { Woodside-Oriakhi et al. } \\
\text { [2011] }\end{array}$ & GA / TS / SA & $2.4 \mathrm{GHz}$ & $31-225$ & 74 to 331 \\
\hline & Di Gaspero et al. [2011] & $\mathrm{LS}+\mathrm{QP}$ & $3.2 \mathrm{GHz}$ & $31-225$ & 2 to 46 \\
\hline & $\begin{array}{l}\text { Golmakani and Fazel } \\
\text { [2011] }\end{array}$ & PSO & $3.0 \mathrm{GHz}$ & $9-150$ & 22 to 40 \\
\hline & Deng et al. [2012] & PSO & $1.6 \mathrm{GHz}$ & $31-225$ & 5 to 76 \\
\hline & Corazza et al. [2013] & $\mathrm{PSO}$ & $2.2 \mathrm{GHz}$ & $50-200$ & 54 to 158 \\
\hline & Cesarone et al. [2013] & MIQP & $2.2 \mathrm{GHz}$ & $31-225$ & 7 to 261 \\
\hline & Lwin and Qu [2013] & $\begin{array}{l}\text { Hybrid } \\
\text { algorithm }\end{array}$ & $3.2 \mathrm{GHz}$ & $31-225$ & 109 to 24823 \\
\hline & $\begin{array}{l}\text { Tuba and Bacanin } \\
{[2014]}\end{array}$ & $\begin{array}{l}\text { Hybrid } \\
\text { algorithm }\end{array}$ & $4.0 \mathrm{GHz}$ & $31-225$ & 12 to 329 \\
\hline & Adebiyi and Ayo [2015] & Modified DE & $4.3 \mathrm{GHz}$ & $31-85$ & 1 to 2 \\
\hline \multirow{5}{*}{ Index Tracking } & Beasley et al. [2003] & $\begin{array}{l}\text { Population } \\
\text { heuristic }\end{array}$ & $225 \mathrm{MHz}$ & $31-225$ & 102 to 942 \\
\hline & Krink et al. [2009] & Hybrid DE & $798 \mathrm{MHz}$ & $65-225$ & 360 to 420 \\
\hline & $\begin{array}{l}\text { Ruiz-Torrubiano and } \\
\text { Suarez [2009] }\end{array}$ & $\mathrm{GA}+\mathrm{QP}$ & $2.0 \mathrm{GHz}$ & $31-225$ & 1 to 47 \\
\hline & $\begin{array}{l}\text { Guastaroba and } \\
\text { Speranza [2012] }\end{array}$ & $\begin{array}{l}\text { Kernel Search } \\
\text { heuristic }\end{array}$ & $2.4 \mathrm{GHz}$ & $31-2151$ & $<5400$ \\
\hline & Scozzari et al. [2013] & $\begin{array}{l}\text { Hybrid DE + } \\
\text { MIQP }\end{array}$ & $2.2 \mathrm{GHz}$ & $65-225$ & 685 to 1080 \\
\hline $\begin{array}{l}\text { Enhanced } \\
\text { Index Tracking }\end{array}$ & $\begin{array}{l}\text { Canakgoz and Beasley } \\
{[2009]}\end{array}$ & $\mathrm{MIP}+\mathrm{EA}$ & $3.0 \mathrm{GHz}$ & $31-497$ & 3.1 (avg.) \\
\hline \multirow{2}{*}{$\begin{array}{l}\text { Credit Risk } \\
\text { Management }\end{array}$} & $\begin{array}{l}\text { Schlottmann and Seese } \\
{[2004]}\end{array}$ & $\begin{array}{l}\begin{array}{l}\text { Hybrid } \\
\text { algorithm }\end{array} \\
\end{array}$ & $2.0 \mathrm{GHz}$ & $25-386$ & 2.2 (avg.) \\
\hline & Lu et al. [2013] & Two-level PSO & $\mathrm{n} / \mathrm{a}$ & $5-100$ & 4 to 12 \\
\hline $\begin{array}{l}\text { Project } \\
\text { Scheduling }\end{array}$ & $\begin{array}{l}\text { Smith-Daniels et al. } \\
{[1996]}\end{array}$ & Heuristic & $\mathrm{n} / \mathrm{a}$ & 230 & 10 \\
\hline \multirow{4}{*}{ Option Pricing } & Kumar et al. [2008] & $\mathrm{ACO}$ & $\mathrm{n} / \mathrm{a}$ & $10-10000$ & 3 to 9 \\
\hline & $\begin{array}{l}\text { Gilli and Schumann } \\
\text { [2012b] }\end{array}$ & $\mathrm{DE} / \mathrm{PSO}$ & $2.5 \mathrm{GHz}$ & $\mathrm{n} / \mathrm{a}$ & 10 to 30 \\
\hline & Sharma et al. [2013] & $\mathrm{PSO}$ & GPU & $20-70$ & $<2$ \\
\hline & Thulasiram et al. [2014] & PSO & GPU & $\mathrm{n} / \mathrm{a}$ & $<9$ \\
\hline \multirow{2}{*}{$\begin{array}{l}\text { Feature } \\
\text { Selection }\end{array}$} & Unler and Murat [2010] & PSO & $2.3 \mathrm{GHz}$ & 17108 & 29 to 155 \\
\hline & Pacheco et al (2009) & TS & $2.4 \mathrm{GHz}$ & 17108 & $<1800$ \\
\hline
\end{tabular}

\section{FUTURE TRENDS REGARDING THE USE OF METAHEURISTICS IN FINANCE}

Our survey sheds light on new emerging issues associated with option pricing, feature selection, bankruptcy prediction, and financial distress prediction are attracting the attention of researchers and practitioners from different communities. Most of these issues have a complex nature and represent rich variants of 
combinatorial optimization problems. The complexity of the aforementioned financial applications requires an increasing use of modern heuristics if near-optimal solutions are needed in reasonable computing times. So far, population-based metaheuristics have been predominantly employed, probably because of their flexibility and the fact that they are supported by a strong community of researchers. This also means that the use of single-solution metaheuristics has been underwhelming, which in our opinion remains an excellent area of research opportunity for researchers in the OR/CS communities. Indeed, these methods tend to be not only efficient but also simpler to configure, since they are less costly in terms of parameter-configuration than population-based approaches.

Also, the existing body of research on financial optimization mainly makes use of exact methods to solve simplified or small-scale models of real-life financial and banking problems. Nevertheless, continuous advances in existing exact and metaheuristic methods, and the fast development of computing techniques and power stimulate scholars to delve deeper into state-of-art methodologies. Indeed, a plethora of novel hybrid methods can now be utilized to resolve rich and real-life financerelated problems. Prominent examples are matheuristics that arise from integration of exact and metaheuristic methods [Doerner and Schmid 2010], or simheuristics [Juan et al. 2015] that result from combination of simulation with metaheuristics. Different works discuss how metaheuristics can be employed to solve optimization problems under uncertainty scenarios [Bianchi et al. 2009, Talbi 2013]. In particular, simheuristics allow to integrate real-life uncertainty both as part of the objective function and as probabilistic constraints in the optimization problems. The ensuing models represent more accurately real-world financial and banking scenarios. Among other strengths, these hybrid methods accommodate elements of: (i) uncertainty (stochastic factors); (ii) dynamism; (iii) diversity of agents and preferences; and (iv) multi-periodicity in financial activities. As solution methods and techniques grow rapidly in complexity, scale and scope, and they can easier find their way in solving more practical instances across a number of fields, a further emergence of financial and banking problems considering complex multi-objective functions and probabilistic constraints is warranted (Figure 3).

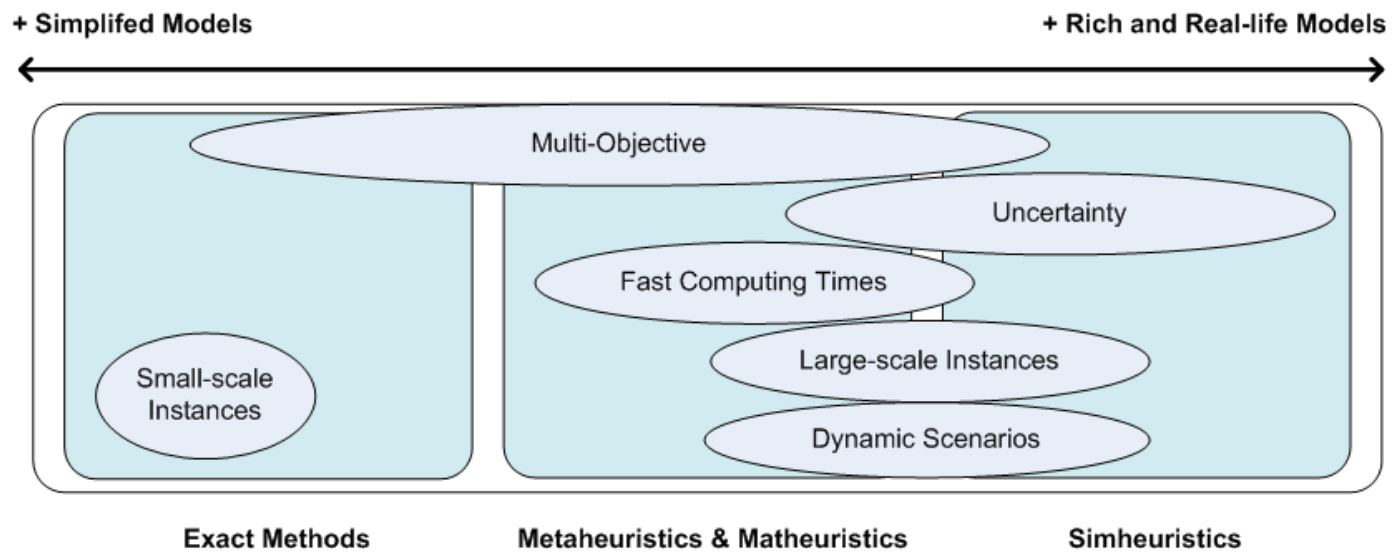

Fig. 3. Trends regarding the use of metaheuristics in finance.

\section{CONCLUSIONS}

In this research, a literature review on the applications of metaheuristics to solve combinatory optimization finance-related problems is presented. The literature 
review covers both classical optimization problems as well as emerging ones. From the analysis of the existing works, several conclusions can be extracted. Firstly, the use of metaheuristics in finance is becoming increasingly more popular among researchers from different communities, as metaheuristics are one of the best alternatives -if not the only one- to deal with rich and real-life optimization problems in the field. Secondly, most of the reviewed works are related either to variants of the portfolio optimization problem or to variants of the credit-risk management problem. Thirdly, it is worth noting that population based metaheuristics have been employed more frequently than single-solution based approaches, which in our opinion represents an opportunity to increase the use of the latter ones in solving these problems. Fourthly, as decision-making processes in finance and banking are growing both in complexity and scale due to financial globalization, there will be an increase in the demand for metaheuristics in order to make efficient decisions. Finally, our research features a section that discusses open opportunities for researchers in the field, as well as future trends regarding the combination of metaheuristics with simulation as a promising way of incorporating real-life uncertainty into the optimization algorithms.

\section{REFERENCES}

A. A. Adebiyi, and C. K. Ayo. 2015. Portfolio Selection Problem Using Generalized Differential Evolution 3. Applied Mathematical Sciences 9, 42 (2015), 2069-2082.

M. Adnan-Aziz, and H. A. Dar. 2006. Predicting corporate bankruptcy: where we stand? Corporate Governance: The International Journal of Business in Society 6, 1 (2006), 18-33.

R. Akbari, V. Zeighami, and K. Ziarati. 2011. Artificial bee colony for resource constrained project scheduling problem. International Journal of Industrial Engineering Computations 2, 1 (2011), 45-60.

F. Allen, and R Karjalainen. 1999. Using genetic algorithms to find technical trading rules. Journal of Financial Economics 51, 2 (1999), 245-271.

M. Aruldoss, M. L. Travis, and V. P. Venkatesan. 2015. A reference model for business intelligence to predict bankruptcy. Journal of Enterprise Information Management 28, 2 (2015), 186-217.

J. E. Beasley. 2013. Portfolio optimisation: models and solution approaches. Tutorials in Operational research 10 (2013), 201-221.

J. E. Beasley, N. Meade, and T. J Chang. 2003. An evolutionary heuristic for the index tracking problem. European Journal of Operational Research 148, 3 (2003), 621-643.

J. M. Berutich, F. López, F. Luna and D. Quintana. 2016. Robust technical trading strategies using GP for algorithmic portfolio selection. Expert Systems with Applications 46, 15 (March 2016), 307-315.

L. Bianchi, M. Dorigo, L. M. Gambardella, and W. J. Gutjah. 2009. A survey on metaheuristics for stochastic combinatorial optimization. Natural Computing 8, 2 (2009), 239-287.

F. Black, and M. Scholes. 1973. The pricing of options and corporate liabilities. The Journal of Political Economy 81, 3 (1973), 637-654.

C. Blum, J. Puchinger, G. R. Raidl, and A. Roli. 2011. Hybrid metaheuristics in combinatorial optimization: A survey. Applied Soft Computing 11, 6 (2011), 4135-4151.

M. A. Boschetti, and V. Maniezzo. 2011. Combining exact methods and heuristics. Wiley Encyclopedia of Operational Research and Management Science (2011).

I. Boussaï, J. Lepagnot, and P. Siarry. 2013. A survey on optimization metaheuristics. Information Sciences 237 (2013), 82-117.

J. Caceres, P. Arias, D. Guimarans, D. Riera, and A. A. Juan. 2014. Rich Vehicle Routing Problem: Survey. ACM Computing Surveys 47, 2 (2014), doi: 10.1145/2666003.

N. A. Canakgoz, and J. E. Beasley. 2009. Mixed-integer programming approaches for index tracking and enhanced indexation. European Journal of Operational Research 196, 1 (2009), 384-399.

S. Casado. 2009. Different metaheuristic strategies to solve the feature selection problem. Pattern Recognition Letters 30, 5-30 (2009), 525-534.

F. Cesarone, A. Scozzari, and F. Tardella. 2013. A new method for mean-variance portfolio optimization with cardinality constraints. Annals of Operational Research 205, 1 (2013), 213-234.

T. J. Chang, N. Meade, J. E. Beasley, and Y. M. Sharaiha. 2000. Heuristics for cardinality constrained portfolio optimisation. Computers \& Operational Research 27, 13 (2000), 1271-1302.

L. Chavez-Bedoya, and J. R. Birge. 2014. Index tracking and enhanced indexation using a parametric approach. Journal of Economics Finance and Administrative Science 19, 36 (2014), 19-44.

N. Chen, B. Ribeiro, A. S. Vieira, J. Duarte, and J. C. Neves. 2011. A genetic algorithm-based approach to cost-sensitive bankruptcy prediction. Expert Systems with Applications 38, 10 (2011), 12939-12945. 
Y. Chen, and X. Wang. 2015. A hybrid stock trading system using genetic network programming and mean conditional value-at-risk. European Journal of Operational Research 240, 3 (2015), 861-871.

S. C. Chiam, K. C. Tan, and A. Al Mamun. 2013. Dynamic index tracking via multi-objective evolutionary algorithm. Applied Soft Computing 13, 7 (2013), 3392-3408.

M. Corazza, G. Fasano, and R. Gusso. 2013. Particle Swarm Optimization with non-smooth penalty reformulation, for a complex portfolio selection problem. Applied Mathematics and Computation 224 (2013), 611-624.

T. Cura. 2009. Particle swarm optimization approach to portfolio optimization. Nonlinear Analysis: Real World Applications 10, 4 (2009), 2396-2406.

V. K. Dabhi, and S. Chaudhary. 2015. Financial Time Series Modeling and Prediction Using Postfix-GP. Computational Economics (2015), 1-35.

G. F. Deng, W. T. Lin, and C. C. Lo. 2012. Markowitz-based portfolio selection with cardinality constraints using improved particle swarm optimization. Expert Systems with Applications 39, 4 (2012), 45584566.

S. Deng, K. Yoshiyama, T. Mitsubuchi, and A. Sakurai. 2015. Hybrid method of multiple kernel learning and genetic algorithm for forecasting short-term foreign exchange rates. Computational Economics 45, 1 (January 2015), 49-89.

U. Derigs, and N. H. Nickel. 2003. Meta-heuristic based decision support for portfolio optimization with a case study on tracking error minimization in passive portfolio management. OR Spectrum 25, 3 (2003), 345-378.

L. Di Gaspero, G. Di Tollo, A. Roli, and A. Schaerf. 2011. Hybrid metaheuristics for constrained portfolio selection problems. Quantitative Finance 11, 10 (2011), 1473-1487.

G. Di Tollo, A. Roli. 2008. Metaheuristics for the portfolio selection problem. International Journal of Operational Research 5, 1 (2008), 13-35.

G. Di Tollo, T. Stützle, and M. Birattari. 2014. A metaheuristic multi-criteria optimisation approach to portfolio selection. Journal of Applied Operational Research 6, 4 (2014), 222-242.

J. Doering, A. A. Juan, R. Kizys, A. Fito, and L. Calvet. 2016. Solving realistic portfolio optimization problems via metaheuristics: A survey and an example. Modeling and Simulation in Engineering, Economics and Management, Vol. 254, p. 22-30. Springer International Publishing.

K. F. Doerner and V. Schmid. 2010. Survey: Matheuristics for rich vehicle routing problems. In Hybrid Metaheuristics, M. Blesa, C. Blum, G. Raidl, A. Roli, and M. Sampels (Eds.). Lecture Notes in Computer Science, Vol. 6373. Springer, 206-221.

P. Dostál. 2013. The use of soft computing for optimization in business, economics and finance. In Metaheuristics Optimization Algorithms in Engineering, Business, Economics and Finance. Pandian M. Vasant (Ed.) IGI Global, 41-86.

L. Dymova, P. Sevastjanov, and K. Kaczmarek. 2016. A Forex trading expert system based on a new approach to the rule-base evidential reasoning. Expert Systems with Applications 51 (1 June 2016), 113.

S. Elhedhli, C. Akdemir, and T. Astebro. 2014. Classification models via Tabu search: An application to early stage venture classification. Expert Systems with Applications 41, 18 (2014), 8085-8091.

B. Fastrich, S. Paterlini, and P. Winker. 2014. Cardinality versus q-norm constraints for index tracking. Quant Finance 14 (2014), 2019-2032

R. Frey, and A. J. McNeil. 2002. VaR and expected shortfall in portfolios of dependent credit risks: conceptual and practical insights. Journal of Banking \& Finance 26, 7 (2002), 1317-1334.

M. Gilli, D. Maringer and P. Winker. 2008. Applications of heuristics in finance. In Handbook on Information Technology in Finance. D. Seese, C. Weinhardt, F. Schlottmann (Eds.) Berlin, Springer, 635-654.

M. Gilli, and E. Schumann. 2012a. Heuristic optimisation in financial modelling. Annals of Operational research 193, 1 (2012), 129-158.

M. Gilli, and E. Schumann. 2012b. Calibrating option pricing models with heuristics. In Natural Computing in Computational Finance. Springer, 9-37.

A. Gogna, and A. Tayal. 2013. Metaheuristics: review and application. Journal of Experimental \& Theoretical Artificial Intelligence 25, 4 (2013), 503-526.

H. R. Golmakani, and M. Fazel. 2011. Constrained portfolio selection using particle swarm optimization. Expert Systems with Applications 38, 7 (2011), 8327-8335.

A. Gorgulho, R. Neves, and N. Horta. 2011. Applying a GA kernel on optimizing technical analysis rules for stock picking and portfolio composition. Expert systems with Applications 38, 11 (2011), 1407214085.

G. Guastaroba, and M. G. Speranza. 2012. Kernel search: an application to the index tracking problem. European Journal of Operational Research 217, 1 (2012), 54-68.

S. Hartmann, and D. Briskorn. 2010. A survey of variants and extensions of the resource-constrained project scheduling problem. European Journal of Operational Research 207, 1 (2010), 1-14.

S. Hartmann, and R. Kolisch. 2000. Experimental evaluation of state-of-the-art heuristics for the resource- 
constrained project scheduling problem. European Journal of Operational Research 127, 2 (2000), 394407.

G. He, and N. J. Huang. 2012. A modified particle swarm optimization algorithm with applications. Applied Mathematics and Computation 219, 3 (2012), 1053-1060.

Z. He, R. Liu, and T. Jia, T. 2012. Metaheuristics for multi-mode capital-constrained project payment scheduling. European Journal of Operational Research 223, 3 (2012), 605-613.

Z. He, N. Wang, and R. Liu. 2015. The Multi-Mode Capital-Constrained Net Present Value Problem. In Handbook on Project Management and Scheduling Vol. 1. Springer, 513-532.

C.-M. Hsu. 2011. A hybrid procedure for stock price prediction by integrating self-organizing map and genetic programming. Expert Systems with Applications 38, 11 (October 2011), 14026-14036.

H. Iba, and T. Sasaki. 1999. Using genetic programming to predict financial data. In Proceedings of the 1999 Congress on Evolutionary Computation. IEEE, doi: 10.1109/CEC.1999.781932.

O. Icmeli, S. Selcuk-Erenguc, and C. J. Zappe. 1993. Project scheduling problems: a survey. International Journal of Operational \& Production Management 13, 11 (1993), 80-91.

I. Iscoe, A. Kreinin, H. Mausser, and O. Romanko. 2012. Portfolio credit-risk optimization. Journal of Banking \& Finance 36, 6 (2012), 1604-1615.

L. Jourdan. 2009. Hybridizing exact methods and metaheuristics: a taxonomy. European Journal of Operational Research 199 (2009), 620-629.

A. A. Juan, J. Faulin, S. E. Grasman, M. Rabe, and G. Figueira. 2015. A review of simheuristics: Extending metaheuristics to deal with stochastic combinatorial optimization problems. Operational Research Perspectives 2 (2015), 62-72

M. A. Kaboudan. 2000. Genetic programming prediction of stock prices. Computational Economics 16, 3 (December 2000), 207-236.

M. Kaucic. 2010. Investment using evolutionary learning methods and technical rules. European Journal of Operational Research 207, 3 (2010), 1717-1727.

M. J. Kim, and I. Han. 2003. The discovery of experts' decision rules from qualitative bankruptcy data using genetic algorithms. Expert Systems with Applications 25, 4 (2003), 637-646.

H.-J. Kim, K.-S. Shin, 2007. A hybrid approach based on neural networks and genetic algorithms for detecting temporal patterns in stock markets. Applied Soft Computing 7, 2 (March 2007), 569-576.

R. Kolisch, and S. Hartmann. 2006. Experimental investigation of heuristics for resource-constrained project scheduling: An update. European journal of operational research 174, 1 (2006), 23-37.

T. Krink, S. Mittnik, and S. Paterlini. 2009. Differential evolution and combinatorial search for constrained index-tracking. Annals of Operational Research 172, 1 (2009), 153-176.

P. R. Kumar, and V. Ravi. 2007. Bankruptcy prediction in banks and firms via statistical and intelligent techniques - A review. European journal of operational research 180, 1 (2007), 1-28.

S. Kumar, R. K. Thulasiram, and P. Thulasiraman. 2008. A bioinspired algorithm to price options. In Proceedings of the 2008 C3S2E conference. ACM, 11-22, doi: 10.1145/1370256.1370260.

K. H. Lee, and G. S. Jo. 1999. Expert system for predicting stock market timing using a candlestick chart. Expert Systems with Applications 16, 4 (1999), 357-364.

Q. Li, and L. Bao. 2014. Enhanced index tracking with multiple time-scale analysis. Economic Modelling 39, 282-292.

Q. Li, L. Sun, and L. Bao. 2011. Enhanced index tracking based on multi-objective immune algorithm. Expert Systems with Applications 38, 5 (2011), 6101-6106.

F. Q. Lu, M. Huang, W. K. Ching, and T. K. Siu. (2013). Credit portfolio management using two-level particle swarm optimization. Information Sciences 237 (2013), 162-175.

S. Luke. 2013. Essentials of Metaheuristics. Lulu, available at http://cs.gmu.edu/ sean/book/metaheuristics/

K. Lwin, and R. Qu. 2013. A hybrid algorithm for constrained portfolio selection problems. Applied intelligence 39, 2 (2013), 251-266.

V. Manahov, R. Hudson, and H. Hoque. 2015. Return predictability and the 'wisdom of crowds': Genetic Programming trading algorithms, the Marginal Trader Hypothesis and the Hayek Hypothesis. Journal of International Financial Markets, Institutions and Money 37 (2015), 85-98.

V. Manahov, R. Hudson, and P. Linsley. 2014. New evidence about the profitability of small and large stocks and the role of volume obtained using Strongly Typed Genetic Programming. Journal of International Financial Markets, Institutions and Money 33 (2014), 299-316.

R. Mansini, W. Ogryczak, and M. G. Speranza. 2014. Twenty years of linear programming based portfolio optimization. European Journal of Operational Research 234, 2 (2014), 518-535.

Y. Marinakis, M. Marinaki, M. Doumpos, N. Matsatsinis, and C. Zopounidis. 2008. Optimization of nearest neighbor classifiers via metaheuristic algorithms for credit risk assessment. Journal of Global Optimization 42, 2 (2008), 279-293.

D. Maringer, and H. Kellerer. 2003. Optimization of cardinality constrained portfolios with a hybrid local search algorithm. Or Spectrum 25, 4 (2003), 481-495.

H. Markowitz. 1952. Portfolio selection*. The journal of finance 7, 1 (1952), 77-91.

H. Mausser, and D. Rosen. 2001. Applying scenario optimization to portfolio credit risk. The Journal of 
Risk Finance 2, 2 (2001), 36-48.

L. Mendes, P. Godinho, and J. Dias. 2012. A Forex trading system based on a genetic algorithm. Journal of Heuristics 18, 4 (2012), 627-656.

R. C. Merton. 1973. Theory of rational option pricing. Bell Journal of Economics and Management Science 4 (1973), 141-183.

K. Metaxiotis, and K. Liagkouras. 2012. Multiobjective evolutionary algorithms for portfolio management: A comprehensive literature review. Expert Systems with Applications 39, 14 (2012), 11685-11698.

J. R. Montoya-Torres, E. Gutierrez-Franco, and C. Pirachican-Mayorga. 2010. Project scheduling with limited resources using a genetic algorithm. International Journal of Project Management 28, 6 (2010), $619-628$.

P. Moradi, and M. Rostami. 2015. Integration of graph clustering with ant colony optimization for feature selection. Knowledge-Based Systems 84 (2015), 144-161.

S. Mousavi, A. Esfahanipour, M. H. F. Zarandi. 2014. A novel approach to dynamic portfolio trading system using multitree genetic programming. Knowledge-Based Systems 66, (August 2014), 68-81.

C. Neely, P. Weller, and R. Dittmar. 1997. Is technical analysis in the foreign exchange market profitable? A genetic programming approach. The Journal of Financial and Quantitative Analysis 32, 4 (1997), $405-426$.

K. J. Oh, T. Y. Kim, and S. Min. 2005. Using genetic algorithm to support portfolio optimization for index fund management. Expert Systems with Applications 28, 2 (2005), 371-379.

S. Oreski, and G. Oreski, G. 2014. Genetic algorithm-based heuristic for feature selection in credit risk assessment. Expert systems with applications 41, 4 (2014), 2052-2064.

M. Ozturk, I. H. Toroslu, and G. Fidan. 2016. Heuristic based trading system on Forex data using technical indicator rules. Applied Soft Computing 43 (June 2016), 170-186.

J. Puchinger, and G. R. Raidl. 2005. Combining metaheuristics and exact algorithms in combinatorial optimization: A survey and classification. In Lecture Notes in Computer Science. Springer, 41-53.

B. Rafaely, and J. A. Bennell. 2006. Optimisation of FTSE 100 tracker funds: A comparison of genetic algorithms and quadratic programming. Managerial Finance 32, 6 (2006), 477-492.

N. Rahmani, V. Zeighami, and R. Akbari. 2015. A study on the performance of differential search algorithm for single mode resource constrained project scheduling problem. Decision Science Letters 4, 4 (2015), 537-550.

G. R. Raidl. 2006. A unified view on hybrid metaheuristics. In Hybrid Metaheuristics. Springer, 1-12.

R. T. Rockafellar, and S. Uryasev. 2000. Optimization of conditional value-at-risk. Journal of risk 2 (2000), 21-42.

R. T. Rockafellar, and S. Uryasev. 2002. Conditional value-at-risk for general loss distributions. Journal of banking \& finance 26, 7 (2002), 1443-1471.

R. Ruiz-Torrubiano, and A. Suarez. 2009. A hybrid optimization approach to index tracking. Annals of Operational Research, 166, 1 (2009), 57-71.

F. Schlottmann, and D. Seese. 2004a. A hybrid heuristic approach to discrete multi-objective optimization of credit portfolios. Computational statistics \& data analysis, 47, 2 (2004), 373-399.

F. Schlottmann, and D. Seese. 2004b. Modern heuristics for finance problems: A survey of selected methods and applications. In Handbook of Computational and Numerical Methods in Finance. Birkhäuser Boston, 331-359.

A. Scozzari, F. Tardella, S. Paterlini, and T. Krink. 2013. Exact and heuristic approaches for the index tracking problem with UCITS constraints. Annals of Operational Research, 205, 1 (2013), 235-250.

A. Serani, M. Diez, E. F. Campana, G. Fasano, D. Peri, and U. Iemma. 2015. Globally convergent hybridization of particle swarm optimization using line search-based derivative-free techniques. In Recent Advances in Swarm Intelligence and Evolutionary Computation. Springer, 25-47.

B. Sharma, R. K. Thulasiram, and P. Thulasiraman. 2013. Normalized particle swarm optimization for complex chooser option pricing on graphics processing unit. The Journal of Supercomputing 66, 1 (2013), 170-192.

K. S. Shin, and Y. J. Lee. 2002. A genetic algorithm application in bankruptcy prediction modeling. Expert Systems with Applications 23, 3 (2002), 321-328.

A. F. Sheta, S. E. M. Ahmed and H. Faris. 2015. Evolving stock market prediction models using multi-gene symbolic regression genetic programming. Artificial Intelligence and Machine Learning (AIML) Journal 15, (December 2015), 11-20.

D. E. Smith-Daniels, R. Padman, and V. L. Smith-Daniels. 1996. Heuristic scheduling of capital constrained projects. Journal of Operational Management 14, 3 (1996), 241-254.

H. Soleimani, H. R. Golmakani, and M. H. Salimi. 2009. Markowitz-based portfolio selection with minimum transaction lots, cardinality constraints and regarding sector capitalization using genetic algorithm. Expert Systems with Applications 36, 3 (2009), 5058-5063.

J. Sun, H. Li, Q. H. Huang, and K. Y. He. 2014. Predicting financial distress and corporate failure: A review from the state-of-the-art definitions, modeling, sampling, and featuring approaches. KnowledgeBased Systems 57 (2014), 41-56. 
E. G. Talbi. 2009. Metaheuristics: from design to implementation. John Wiley \& Sons.

E. G. Talbi. 2013. Metaheuristics in Uncertain Environments. Wiley Publishing.

R. K. Thulasiram, P. Thulasiraman, H. Prasain, and G. K. Jha. 2014. Nature-inspired soft computing for financial option pricing using high-performance analytics. Concurrency and Computation: Practice and Experience (2014), doi: 10.1002/cpe.3360

M. Tuba, and N. Bacanin. 2014. Artificial Bee Colony Algorithm Hybridized with Firefly Algorithm for Cardinality Constrained Mean-Variance Portfolio Selection Problem. Appl. Math 8, 6 (2014), 28312844.

A. Unler, and A. Murat. 2010. A discrete particle swarm optimization method for feature selection in binary classification problems. European Journal of Operational Research 206, 3 (2010), 528-539.

F. Varetto. 1998. Genetic algorithms applications in the analysis of insolvency risk. Journal of Banking \& Finance 22, 10 (1998), 1421-1439.

M. Woodside-Oriakhi, C. Lucas, and J. E. Beasley. 2011. Heuristic algorithms for the cardinality constrained efficient frontier. European Journal of Operational Research 213, 3 (2011), 538-550.

C. Wu, X. Wang, and J. Lin. 2014. Optimizations in Project Scheduling: A State-of-Art Survey. In Optimization and Control Methods in Industrial Engineering and Construction. Springer, 161-177.

B. Xue, M. Zhang, and W. N. Browne. 2014. Particle swarm optimisation for feature selection in classification: Novel initialisation and updating mechanisms. Applied Soft Computing 18 (2014), 261276.

Y. Yamai, and T. Yoshiba. 2005. Value-at-risk versus expected shortfall: A practical perspective. Journal of Banking \& Finance 29, 4 (2005), 997-1015.

X. S. Yang. 2010. Nature-inspired metaheuristic algorithms. Luniver Press.

Z. Yin, A. Brabazon, and C. O'Sullivan. 2007. Adaptive genetic programming for option pricing. In Proceedings of the 9th annual conference companion on Genetic and evolutionary computation. ACM, 2588-2594.

J. Zhou, P. E. Love, X. Wang, K. L. Teo, and Z. Irani. 2013. A review of methods and algorithms for optimizing construction scheduling. Journal of the Operational Research Society 64, 8 (2013), 10911105.

C. Zopounidis. 2015. Predicting Credit Ratings Using a Robust Multicriteria Approach. Quantitative Financial Risk Management: Theory and Practice (2015), 312-334. 